\title{
A simple and robust way towards reversible mechanochromism: using liquid crystal elastomer as a mask
}

\author{
Zhijian Wang1), Wei Fan'2), Qiguang $\mathrm{He}^{1)}$, Yang Wang3), Xudong Liang'), Shengqiang \\ $\mathrm{Cai}^{1,2)^{*}}$ \\ ${ }^{1}$ Department of Mechanical and Aerospace Engineering, University of California, San Diego, La Jolla, CA \\ 92093, USA \\ ${ }_{2}^{2}$ Materials Science and Engineering Program, University of California, San Diego, La Jolla, CA 92093, \\ USA \\ 3 Department of Structural Engineering, University of California, San Diego, La Jolla, CA 92093, USA
}

\begin{abstract}
Mechanochromism is color change of a material induced by external force. Recently, it has been intensively studied for its potential applications in biomimetic camouflage devices and strain sensors. In this article, we developed a simple and robust way to fabricate reversible mechanochromic trilayer system using liquid crystal elastomer (LCE) and transparent elastomers. Subjected to mechanical stretch, an opaque polydomain LCE can transform to transparent monodomain, which is used as a mask to block (without stretch) or show (with stretch) the color patterns painted on an elastomeric layer attached underneath. In this article we also demonstrated the visualization of heterogeneous strain field in the developed mechanochromic system.
\end{abstract}

* Corresponding author

E-mail: shqcai@ucsd.edu (Shengqiang Cai) 


\section{Introduction}

Color change is one of the most important responses to external stimuli in various biological systems [1]. For example, cephalopods show various color patterns for camouflage by contracting different muscle fibers attached to pigment-containing cells in the skin $[2,3]$. Inspired by this intriguing capability of biological systems, researchers have been trying to synthesize different material systems which can also change color when subjected to external forces, which is often called mechanochromism [4-8]. For instance, by dispersing dye bis(benzoxazolyl)stilbene into polyester [poly(1,4-butylene succinate)], Pucci et al. fabricated polymer blend film which could change its light emission from green to blue when the applied uniaxial strain was larger than $50 \%$ [9]. In addition to simply dispersing dyes into polymer matrix, several functional groups such as spiropyran [10-15], rhodamine [16], diarylbibenzofuranone unit $[17,18]$ and dioxetane unit $[19,20]$ have been synthesized and covalently incorporated into polymer backbone. Those functional groups can emit fluorescent light when subjected to forces above certain level, which makes the synthesized polymer be mechanochromic material. Compared to the dye dispersion system, the polymers with integrated functional groups on their backbones show much higher spatial resolution of strain field [2O,21]. However, complex synthetic steps are often required to obtain those functional groups. In addition, for a specific functional group, it can only emit light with certain range of wavelength, which limits its applications in some devices where a full and adjustable light spectrum is desired. In this article, using liquid crystal elastomer (LCE) as a mechanically switchable mask, we propose to develop a simple but robust method to fabricate the mechanochromic trilayer system.

LCE can be formed by a combination of liquid crystal molecules and polymer network [22-24]. Synthesized LCE is usually polydomain when the environmental 
temperature is below isotropic clearing temperature (Figure 1). The domain size inside a LCE is typically several hundreds of nanometers, which makes polydomain LCE strongly scatter light and appear opaque [25]. Polydomain LCE can transform to mondomain and become transparent due to the alignment of the liquid crystal mesogens [26]. Based on this simple and robust mechanical-optical coupling effect, in this study we developed a mechanochromic sandwich-like structure composed of one LCE layer and two VHB layers as shown in Figure 2(a). In the trilayer structure, polydomain LCE, acting as a mask, was sandwiched between two VHB layers. The bottom VHB layer was painted with dyes in desired patterns, while the top VHB layer served as an additional supporting layer. When the whole trilayer structure was stretched beyond a certain value, polydomain LCE became transparent and the pattern painted on the VHB layer became visible.

\section{Materials and methods.}

\subsection{Materials}

(1,4-bis-[4-(3-acryloyloxypropyloxy) benzoyloxy]-2-methylbenzene) (RM257) (Wilshire company, 95 \%), (2-hydroxyethoxy)-2-methylpropiophenone (HHMP, SigmaAldrich, 98\%), 2,2'-(ethylenedioxy) diethanethiol (EDDET, Sigma-Aldrich, 95\%), pentaerythritol tetrakis (3-mercaptopropionate) (PETMP, Sigma-Aldrich, 95\%), dipropylamine (DPA, Sigma-Aldrich, 98\%) and VHB film (3M company) were used as received without further purification.

2.2 Synthesis of LCE film

The LCE was prepared following the procedures reported previously [27,28]. Figure 1 sketched the preparation route of the LCE film used in the current study. Firstly, the diacrylate mesogen RM257 was dissolved in toluene and the mixture was heated at 
$85^{\circ} \mathrm{C}$ to be homogenous. Then, HHMP, the crosslinking initiator in the second polymerization stage, was added into the solution and heated to be dissolved. After that, EDDET, the flexible spacer in the LCE and PETMP, the tetra-arm thiol crosslinker, were added into the stirring mixture dropwisely. Then the Michael addition reaction catalyst DPA was added later. The mixture was degassed in the vacuum to remove the bubbles inside and poured into a rectangular glass mold with the thickness of $1 \mathrm{~mm}$. The Michael addition reaction was taken in the room temperature overnight. In this step, the thiol groups in EDDET and PETMP would react with the acrylate groups in RM257 via Michael addition reaction to form the first crosslinking network. Then after putting into the oven to evaporate the toluene for $6 \mathrm{~h}$, the film was placed under $365 \mathrm{~nm}$ UV light (UVP High-Intensity UV Inspection Lamp, $100 \mathrm{~W}$ ) for $15 \mathrm{~min}$. The added acrylate group amount in RM257 is excessive compared to the thiol groups, thus the unreacted acrylate groups after first crosslinking step can undergo the second crosslinking step by the photoinitiation of HHMP. The second crosslinking density can be easily controlled by tuning the amount of excessive RM257. In this work, three LCE samples with different excessive RM257 ratios (1\%, 5\% and 15\%) were prepared. $1 \%, 5 \%$ and $15 \%$ here represent the excessive amount of acrylate groups in RM257 compared to the added thiol groups. The film obtained was opaque in the sunlight which works as an excellent candidate for the cover of the patterned and colored VHB layer.

2.3 Fabrication of mechanochromic trilayer film

Figure 2(a) shows the fabrication process of a mechanochromic trilayer film. The bottom VHB layer is sprayed with the Rhodamine B (RhB), a well-known fluorescent dye which has been well studied as fluorescent probes for its excellent optical properties [2931]. The as-prepared LCE layer, which is polydomain and appears opaque, is attached to the VHB layer. Another VHB layer is covered onto the LCE film, acting as an additional supporting layer. The whole trilayer film appears as opaque in free standing state. 
2.4 Measurement of mechanical and optical properties of LCE film

Stress-strain curves of LCE films under uniaxial tensile stress were measured using Instron Machine (5965 Dual Column Testing systems, Instron) with a $1000 \mathrm{~N}$ loading cell. Both ends of the samples were glued onto acrylic plates which were clamped by the grippers of Instron Machine. The engineering strain rate was set to be $1 \mathrm{~min}^{-1}$ in the tests.

The transmittance measurement of LCE for different light wavelength was conducted on the UNICO $4802 \mathrm{UV}-\mathrm{Vis}$ double beam spectrophotometer.

\section{Results and discussion}

Figure 2(b) shows the mechanochromism of the fabricated trilayer system. In free standing state, the trilayer film was white and opaque in sunlight. When we uniaxially stretched the film, red color of the dye (RhB) gradually appeared. Such mechanochromism is associated with the polydomain-to-monodomain transition in the middle LCE layer induced by stretch. As explained above, polydomain LCE is opaque while monodomain one is transparent. The color painted on the bottom VHB layer becomes visible after the middle LCE layer becomes transparent.

We next tested the response of the developed mechanochromic system under UV light (365 nm). As shown in Figure 2(c), in free standing state, the trilayer film appeared bluish because of the reflection of UV light, which indicates that UV light couldn't pass through the polydomain LCE layer. When the film was stretched, the fluorescent dye (RhB) painted on the bottom VHB layer emitted very strong fluorescence, indicating that UV light can pass through stretched LCE layer and excite the dyes when LCE layer changed into monodomain after stretching. It is well known that fluorescent light can be easily distinguished from background light noise, and thus more easily to be detected. In the following experiments, we only focused on the change of fluorescence of the 
fabricated mechanochromic film subjected to stretch. We hope the demonstration of the response of the developed mechanochromic film in both UV light and sunlight could broaden its potential applications.

To obtain a mechanochromic film with good stretchability and fast response, we first measured stress-strain curves of LCE films with different crosslinking densities. As described in section 2, LCE film was prepared following the two-stage thiol-acrylate Michael addition and polymerization procedures reported previously [27,28]. Crosslinking density of the LCE film can be easily controlled by varying the amount of RM257 as discussed in section 2. Figure 3(a) shows the stress-strain curves of the LCE films with different crosslinking density. It can be seen that the tensile strength of the LCE film is increased from 1.7 MPa to 2.9 MPa with the excessive RM257 amount increased from $1 \%$ to $15 \%$, while the maximum strain of LCE film is decreased from $250 \%$ to $90 \%$.

We next conducted the recovery experiments of LCE films with different crosslinking densities. In the experiment, a LCE film was uniaxially stretched by 2 times of its original length and then released to allow it recover to its original state. The time for its recovery to the initial length was defined as the recovery time in the article. We found that the LCE film with $1 \%$ excessive RM257 was highly stretchable but the recovery time can be as long as several hours, while it took only about $4 \mathrm{~min}$ for stretched LCE film with $5 \%$ excessive RM257 to recover to its initial state. A stretched LCE film with $15 \%$ excessive RM257 can recover even faster, however it is much less stretchable and its stretch-induced transmittance change is less significant. The response time of the developed mechanochromic system can be important for its applications, which has been rarely studied, or at least reported, in other mechanochromic materials. In the current study, the LCE film with 5\% excessive RM257 was chosen as the middle mask layer in our mechanochromic system. 
We then tested the mechanical properties of the entire trilayer mechanochromic film. Figure 3(b) plots the stress-strain curves of a VHB film, a LCE film (with $5 \%$ excessive RM257) and the entire trilayer film, respectively. We found that the VHB film is much more compliant and stretchable than the LCE film. Consequently, the stretchability of the trilayer film is mainly determined by the stretchability of the middle LCE layer. Although the VHB film is very transparent and has negligible influence on the optical property of the entire trilayer mechanochromic film, VHB film can strongly affect its response time. As shown in the supplementary video s1, it took only 30 seconds for a trilayer film to recover to its original length, compared to 4 min recovery time for the middle LCE layer.

To quantitatively study light transmittance of polydomain LCE film under different levels of stretch, UV-Vis spectra measurement of LCE film with different strains was conducted. Figure 4(a) shows the light transmittance of polydomain LCE films with different applied stretches. It can be clearly seen that polydomain LCE film can almost fully block the light in the full spectrum in its initial opaque state with no stretch. When the applied uniaxial strain is $90 \%$, visible light can partially pass through the LCE film. The LCE film allows the pass of light with the full range of wavelength in our tests when the applied strain exceeds $110 \%$. The light transmittance of LCE changed dramatically when the strain is around 100\% (Figure 4(b)). At this point, polydomain LCE switches to monodomain, which is consistent with the plateau observed in the stress-strain curve as shown in Figure 3(a). As sketched in Figure 4(c), in polydomain state, liquid crystal molecules align well in one domain. But the orientation of liquid crystal molecules in one domain is different from another, which can cause strong light scattering. In monodomain state, liquid crystal molecules align uniformly in the entire material, which makes it transparent. The light transmittance property of LCE makes it possible to be a mechanically switchable light mask for various dyes painted on the bottom VHB layer, 
varying from non-fluorescent dyes to fluorescent dyes. We would also like to point out that it is difficult to make the LCE to be sensitive to small strain due to the relative large critical strain for polydomain to monodomain transition. However, the critical strain is adjustable to certain extent when synthetic condition of LCE is slightly changed.

To study the light transmittance variation of the LCE film subjected to cyclic loading, we repeatedly stretched the LCE film to two times of its initial length and released the force to allow the film to recover back to its original length. We measured the transmittance of the LCE film in its undeformed state and in the state of maximal deformation for the light with the wavelength of $550 \mathrm{~nm}$. The results are shown in Figure 5. The transmittance variation of the LCE film subjected to cyclic loading almost maintain unchanged, which indicates that the LCE film is a robust mechanically tunable mask for cyclic loading.

LCE can also transit from opaque polydomain to transparent amorphous state upon heating above the isotropic clearing temperature $[23,25]$. Such temperature sensitivity of LCE can also be used to make the developed mechanochromic film respond to temperature variation. In the experiment, we sprayed 'UCSD' characters using RhB to the bottom VHB layer. As shown in Figure 6, at room temperature, the mechanochromic film is white. Upon heating above the isotropic clearing temperature $\left(80^{\circ} \mathrm{C}\right)$, the UCSD character becomes visible and the mechanochromic film recover to the initial white state after cooling down to the room temperature. The thermally induced transparency change of LCE is associated with phase transition of liquid crystal molecules upon heating. Above the isotropic clearing temperature, liquid crystal molecules transit from nematic phase to amorphous phase which is transparent. Therefore, the thermally induced transparency change of LCE film is different from the stretch-induced transparency change which is due to the polydomain-monodomain transition as discussed previously. Compared to most mechanochromic film, dual-sensitivity of the trilayer film developed 
in the article is unique and can be potentially useful as a combined strain and temperature sensor.

As shown in the previous experiments, mechanoresponsive behavior of the fabricated trilayer mechanochromic system is highly sensitive to applied strain. To further illustrate this feature, we fabricated a tricolor trapezoid film with red (RhB), orange (mixed color of $\mathrm{RhB}$ and $\mathrm{Rh} 6 \mathrm{G}$ ) and yellow (Rh6G) color stripes (Figure 7). As a rough estimate, the tensile stress in the film is $\sigma=F / A$, where $F$ is the tensile force and $A$ is cross sectional area. Because the force is a constant in the entire film, the tensile stress is large in the narrow region which consequently results in large deformation. When the film is stretched with the average stretch larger than 1.5, the yellow area on the top of the film first appears; further stretching the film with average stretch above 1.75 leads to the appearance of both yellow and orange areas; further increasing the stretch ratio to 2 results in the appearance of the entire tricolor pattern. By observing the patterns showing on the mechanochromic film, we could easily estimate its average strain.

In the last experiment, by applying uniaxial stretch onto the fabricated mechanochromic film with periodically distributed square holes, we demonstrated that a complex visible pattern can also be developed in the film, which is very similar to the pattern of its heterogeneous stretch field. As shown in Figure 8(a), when the mechanochromic film with periodically distributed square holes is stretched uniaxially, due to stretch concentration, red fluorescent color first appears near the edge of the holes. Further stretch of the film leads to a continuous variation of the pattern in the film. Using ABAQUS (www.simulia.com), we conducted FEM simulation to compute the stretch field in the film. Figure 8(b) plots the distribution of the first variant of CauchyGreen deformation tensor $I=\left(1+\varepsilon_{1}\right)^{2}+\left(1+\varepsilon_{2}\right)^{2}+\left(1+\varepsilon_{3}\right)^{2}$, where $\varepsilon_{1}, \varepsilon_{2}, \varepsilon_{3}$ are the principal strains in the film. Cauchy-Green deformation tensor has been commonly used to 
characterize stretching state of a material $[32,33]$. The correlation between the stretch field and the visible colored pattern is obvious.

\section{Concluding Remarks}

In this article, we developed a simple method to fabricate trilayer mechanochromic film, which is composed of one liquid crystal elastomer layer sandwiched by two VHB layers. The trilayer film can change its color through mechanical stretching and heating, and the color change disappears in a short time when stretch is released or the film is cooled down. The color change in the trilayer film can be observed clearly either under sunlight or UV light, which expands the application of the LCE-based mechanochromic film. We have also demonstrated that the developed mechanochromic film shows visible patterns closely correlated to its heterogeneous stretch field.

\section{Acknowledgement}

The work is supported by the National Science Foundation through Grant No. CMMI1554212. 


\section{Reference}

[1] Morin, S. A.; Shepherd, R. F.; Kwok, S. W.; Stokes, A. A.; Nemiroski, A.; Whitesides, G. M. Camouflage and display for soft machines. Science 2012, 337 (6096), 828-832.

[2] Hanlon, R. Cephalopod dynamic camouflage. Current Biology 2oo7, 17 (11), R400-R404.

[3] Hanlon, R.; Chiao, C.-C.; Mäthger, L.; Barbosa, A.; Buresch, K.; Chubb, C. Cephalopod dynamic camouflage: bridging the continuum between background matching and disruptive coloration. Philosophical Transactions of the Royal Society of London B: Biological Sciences 2009, 364 (1516), 429-437.

[4] Caruso, M. M.; Davis, D. A.; Shen, Q.; Odom, S. A.; Sottos, N. R.; White, S. R.; Moore, J. S. Mechanically-induced chemical changes in polymeric materials. Chem. Rev. 2009, 109 (11), 5755-5798.

[5] Dal Molin, M.; Verolet, Q.; Colom, A.; Letrun, R.; Derivery, E.; Gonzalez-Gaitan, M.; Vauthey, E.; Roux, A.; Sakai, N.; Matile, S. Fluorescent Flippers for Mechanosensitive Membrane Probes. J. Am. Chem. Soc. 2o15, 137 (2), 568-571.

[6] Dal Molin, M.; Verolet, Q.; Soleimanpour, S.; Matile, S. Mechanosensitive membrane probes. Chem. Eur. J. 2015, 21 (16), 6012-6021.

[7] Ciardelli, F.; Ruggeri, G.; Pucci, A. Dye-containing polymers: methods for preparation of mechanochromic materials. Chem. Soc. Rev. 2013, 42 (3), 857-870.

[8] Balkenende, D. W.; Coulibaly, S.; Balog, S.; Simon, Y. C.; Fiore, G. L.; Weder, C. Mechanochemistry with metallosupramolecular polymers. J. Am. Chem. Soc. 2014, 136 (29), 10493-10498.

[9] Pucci, A.; Di Cuia, F.; Signori, F.; Ruggeri, G. Bis (benzoxazolyl) stilbene excimers as temperature and deformation sensors for biodegradable poly (1, 4-butylene succinate) films. J. Mater. Chem. 2oo7, 17 (8), 783-790. 
[10] Davis, D. A.; Hamilton, A.; Yang, J.; Cremar, L. D.; Van Gough, D.; Potisek, S. L.;

Ong, M. T.; Braun, P. V.; Martínez, T. J.; White, S. R. Force-induced activation of covalent bonds in mechanoresponsive polymeric materials. Nature 2oo9, 459 (7243), 68-72.

[11] Lee, C. K.; Davis, D. A.; White, S. R.; Moore, J. S.; Sottos, N. R.; Braun, P. V. Force-induced redistribution of a chemical equilibrium. J. Am. Chem. Soc. 2010, 132 (45), 16107-16111.

[12] O’Bryan, G.; Wong, B. M.; McElhanon, J. R. Stress sensing in polycaprolactone films via an embedded photochromic compound. ACS Appl. Mater. Interfaces 2010, 2 (6), 1594-1600.

[13] Fang, X.; Zhang, H.; Chen, Y.; Lin, Y.; Xu, Y.; Weng, W. Biomimetic modular polymer with tough and stress sensing properties. Macromolecules 2013, 46 (16), 65666574 .

[14] Peterson, G. I.; Larsen, M. B.; Ganter, M. A.; Storti, D. W.; Boydston, A. J. 3DPrinted Mechanochromic Materials. ACS Appl. Mater. Interfaces 2o14, 7 (1), 577-583.

[15] Gossweiler, G. R.; Brown, C. L.; Hewage, G. B.; Sapiro-Gheiler, E.; Trautman, W. J.; Welshofer, G. W.; Craig, S. L. Mechanochemically Active Soft Robots. ACS Appl. Mater. Interfaces 2015, 7 (40), 22431-22435.

[16] Wang, Z.; Ma, Z.; Wang, Y.; Xu, Z.; Luo, Y.; Wei, Y.; Jia, X. A Novel Mechanochromic and Photochromic Polymer Film: When Rhodamine Joins Polyurethane. Adv. Mater. 2015, 27 (41), 6469-6474.

[17] Imato, K.; Nishihara, M.; Kanehara, T.; Amamoto, Y.; Takahara, A.; Otsuka, H. Self- Healing of Chemical Gels Cross- Linked by Diarylbibenzofuranone- Based Trigger- Free Dynamic Covalent Bonds at Room Temperature. Angew. Chem. Int. Ed. 2012, 51 (5), 1138-1142. 
[18] Imato, K.; Kanehara, T.; Ohishi, T.; Nishihara, M.; Yajima, H.; Ito, M.; Takahara, A.; Otsuka, H. Mechanochromic Dynamic Covalent Elastomers: Quantitative Stress Evaluation and Autonomous Recovery. ACS Macro Letters 2015, 4 (11), 1307-1311.

[19] Chen, Y.; Spiering, A.; Karthikeyan, S.; Peters, G. W.; Meijer, E.; Sijbesma, R. P. Mechanically induced chemiluminescence from polymers incorporating a 1, 2-dioxetane unit in the main chain. Nat. Chem. 2012, 4 (7), 559-562.

[20] Ducrot, E.; Chen, Y.; Bulters, M.; Sijbesma, R. P.; Creton, C. Toughening elastomers with sacrificial bonds and watching them break. Science 2014, 344 (6180), 186-189.

[21] Clough, J. M.; Creton, C.; Craig, S. L.; Sijbesma, R. P. Covalent Bond Scission in the Mullins Effect of a Filled Elastomer: Real- Time Visualization with Mechanoluminescence. Adv. Funct. Mater. 2016. DOI: 10.1002/adfm.201602490.

[22] Xie, P.; Zhang, R. Liquid crystal elastomers, networks and gels: advanced smart materials. J. Mater. Chem. 2oo5, 15 (26), 2529-2550.

[23] Ohm, C.; Brehmer, M.; Zentel, R. Liquid crystalline elastomers as actuators and sensors. Adv. Mater. 2010, 22 (31), 3366-3387.

[24] White, T. J.; Broer, D. J. Programmable and adaptive mechanics with liquid crystal polymer networks and elastomers. Nat. Mater. 2015, 14 (11), 1087-1098.

[25] Warner, M.; Terentjev, E. M. Liquid crystal elastomers. OUP Oxford: 2003; Vol. 120.

[26] Kundler, I.; Finkelmann, H. Strain- induced director reorientation in nematic liquid single crystal elastomers. Macromol. Rapid Commun. 1995, 16 (9), 679-686.

[27] Ahn, C.; Liang, X.; Cai, S. Inhomogeneous stretch induced patterning of molecular orientation in liquid crystal elastomers. Extreme Mechanics Letters 2015, 5, 30-36. 
[28] Yakacki, C.; Saed, M.; Nair, D.; Gong, T.; Reed, S.; Bowman, C. Tailorable and programmable liquid-crystalline elastomers using a two-stage thiol-acrylate reaction. RSC Advances 2015, 5 (25), 18997-19001.

[29] Fölling, J.; Belov, V.; Kunetsky, R.; Medda, R.; Schönle, A.; Egner, A.; Eggeling, C.; Bossi, M.; Hell, S. e. W. Photochromic rhodamines provide nanoscopy with optical sectioning. Angew. Chem. 2007, 119 (33), 6382-6386.

[30] Dickinson, B. C.; Huynh, C.; Chang, C. J. A palette of fluorescent probes with varying emission colors for imaging hydrogen peroxide signaling in living cells. J. Am. Chem. Soc. 2010, 132 (16), 5906-5915.

[31] Kubin, R. F.; Fletcher, A. N. Fluorescence quantum yields of some rhodamine dyes. J. Lumin. 1983, 27 (4), 455-462.

[32] Wang, Q.; Gossweiler, G. R.; Craig, S. L.; Zhao, X. Cephalopod-inspired design of electro-mechano-chemically responsive elastomers for on-demand fluorescent patterning. Nat. Commum. 2014, 5, 4899.

[33] Silberstein, M. N.; Cremar, L. D.; Beiermann, B. A.; Kramer, S. B.; Martinez, T. J.; White, S. R.; Sottos, N. R. Modeling mechanophore activation within a viscous rubbery network. J. Mech. Phys. Solids 2014, 63, 141-153. 

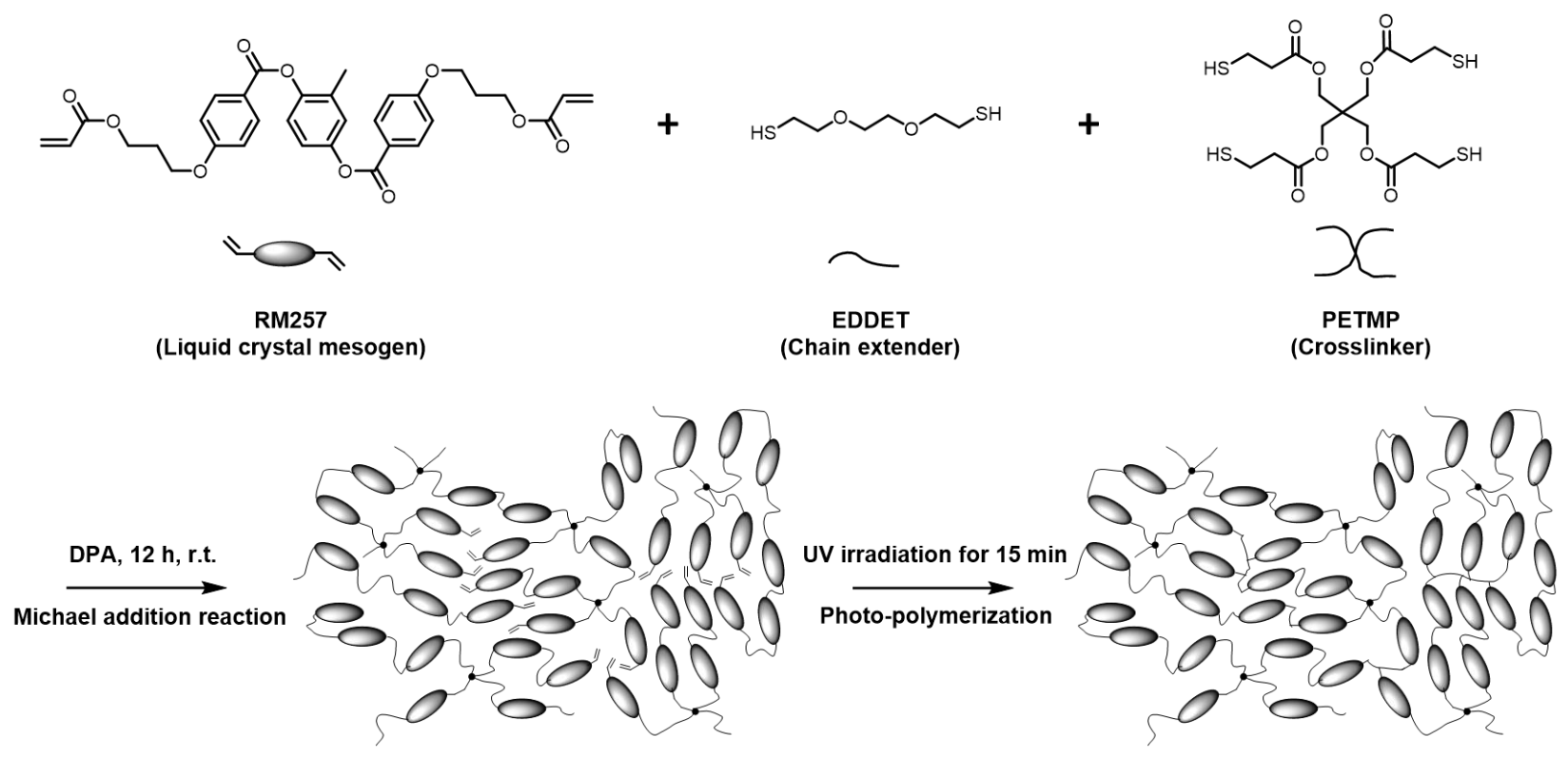

Figure 1. Schematic of the synthesis route of polydomain LCE film. 
(a)

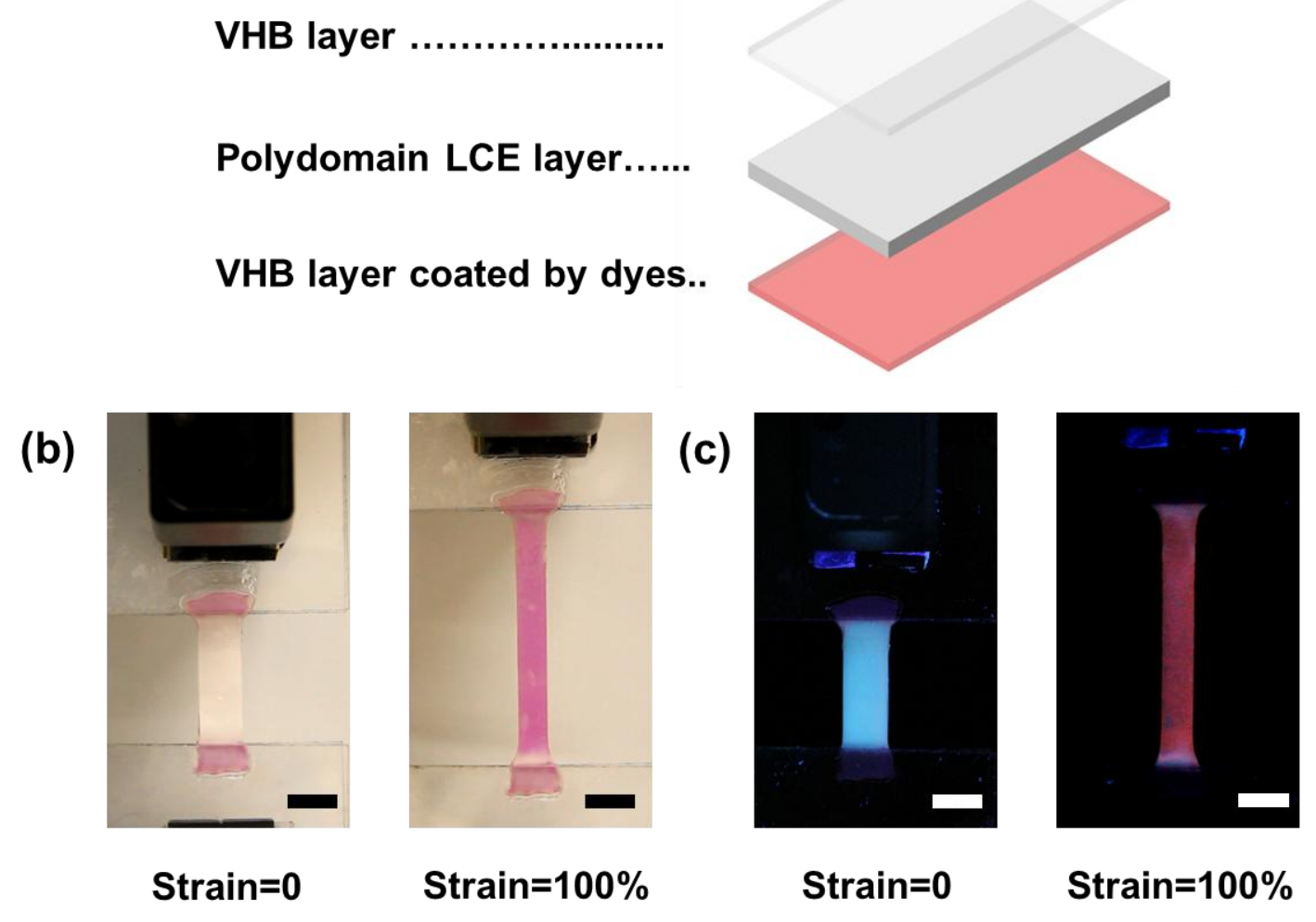

Figure 2. (a) The schematic of the fabricated trilayer mechanochromic film. (b) The fabricated mechanochromic film changed its color from white to red in sunlight, when it was uniaxially stretched beyond $100 \%$ strain. (c) Under the illumination of UV light, the film changed from bluish to red fluorescent light. Scale bar in the figure is $1 \mathrm{~cm}$. 

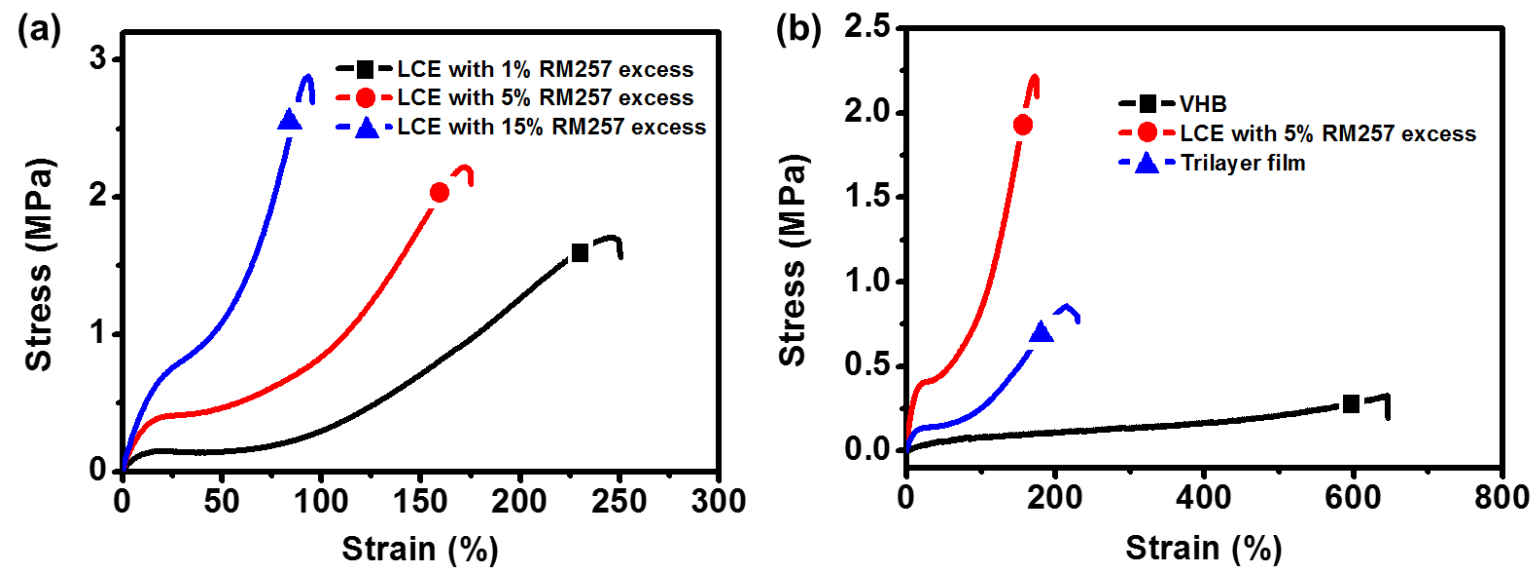

Figure 3. (a) Stress-strain curves of LCE film with different RM257 content. With the increase of the RM257 content(the crosslinking density in the second crosslinking step), the modulus of the LCE increases greatly but the stretchability decreases. (b) Stressstrain curves of VHB, LCE and VHB/LCE/VHB trilayer film. The LCE film with $5 \%$ RM257 excess was chosen for fabricating the mechanochromic film in the experiment. 
(a)

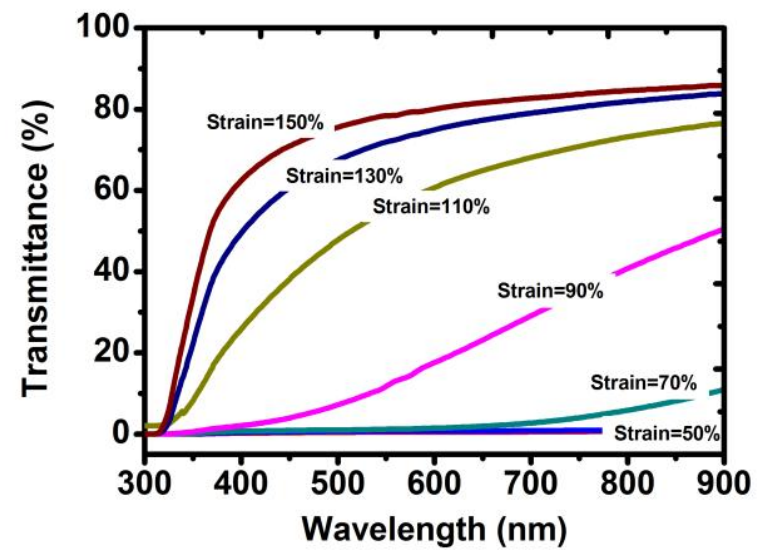

(b)

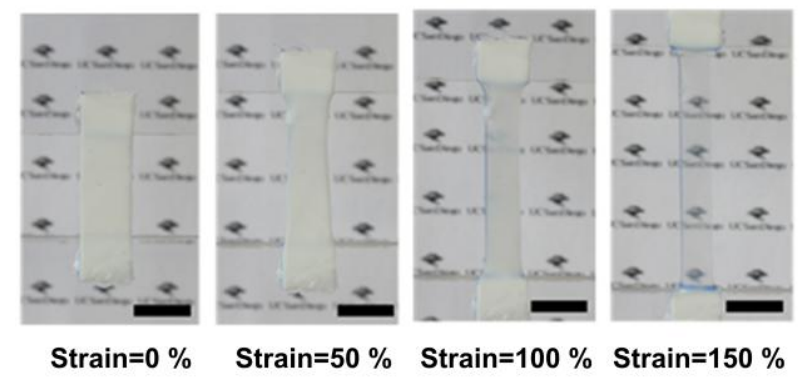

(c)

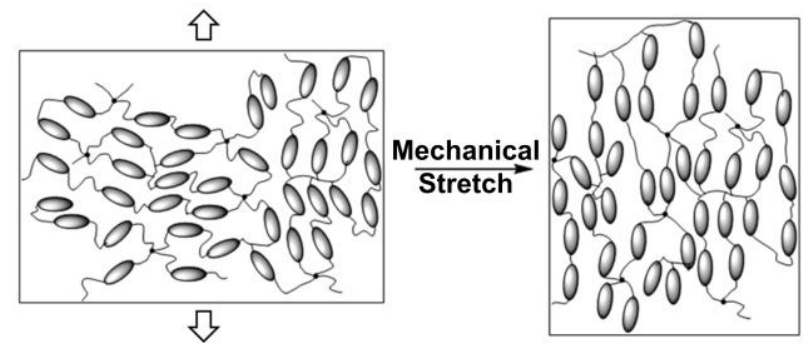

Polydomain state

(Opaque)

Monodomain state (Transparent)

Figure 4. (a) Transmittance spectra of a polydomain LCE film subjected to different tensile strains. When the strain was less than $70 \%$, the film could almost fully block the light for a wide range of wavelength and it became transparent for the visible light when strain was larger than $110 \%$. (b) Optical images of polydomain LCE film subjected to different tensile strains. Scale bar: $1 \mathrm{~cm}$. (c) Schematics of stretch induced polydomainto-monodomain tranisition in a liquid crystal elastomer. In polydomain state, liquid crystal molecules align well in one domain. But the orientation of liquid crystal molecules in one domain is different from another, which can cause strong light 
scattering. In monodomain state, liquid crystal molecules align uniformly in the entire material, which makes it transparent.

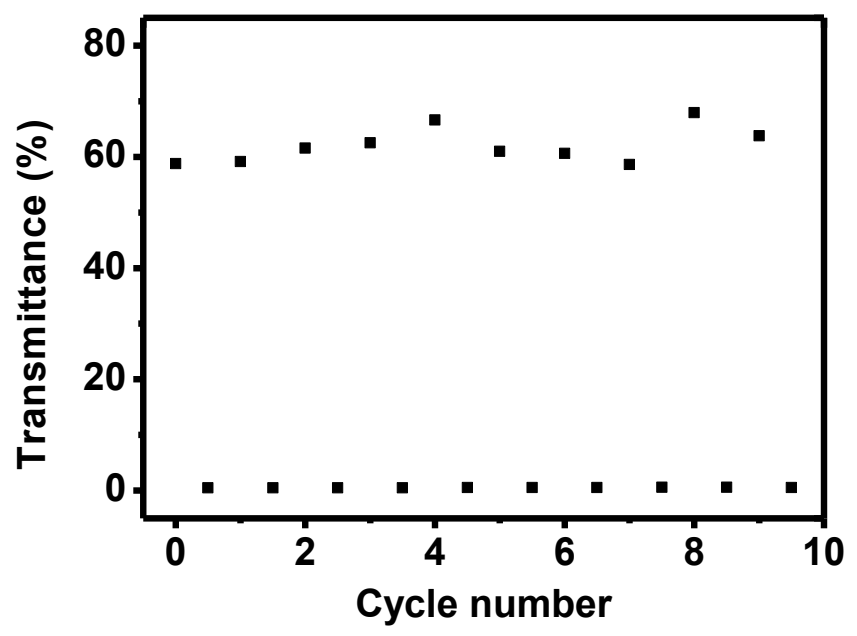

Figure 5. Transmittance change of a polydomain LCE film for the light with 550nm wavelength during cyclic loading and unloading. In each cycle, the LCE film was stretched to 2 times of its initial length and then released to allow it fully recover to the initial length. The maximum transmittance of the film was achieved when the stretch is two, and the minimum transmittance was achieved when there is no stretch in the film. 

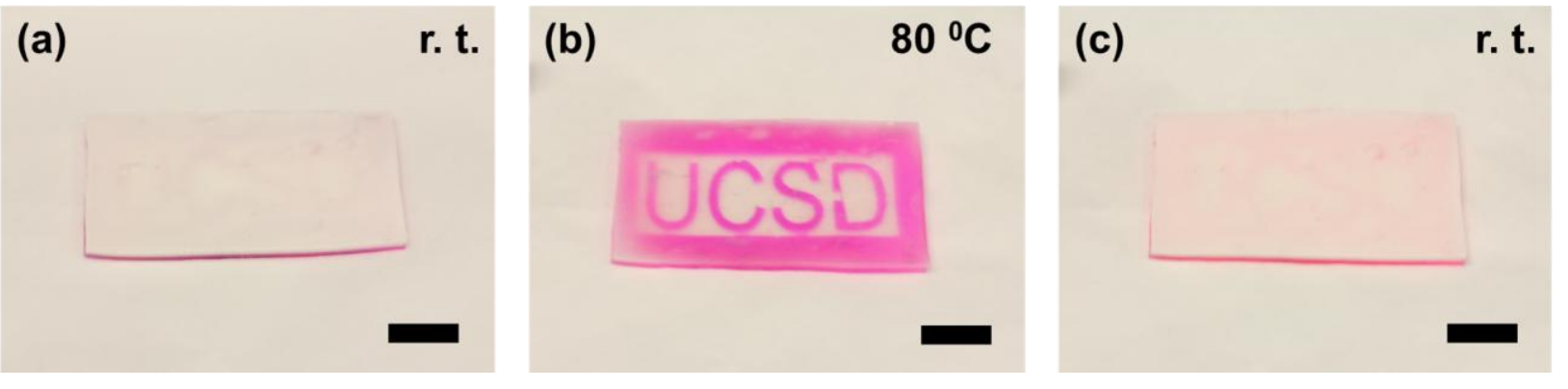

Figure 6. Thermal response of the fabricated mechanochromic trilayer film. (a) At room temperature, the film was white. (b) When the trilayer film was heated up above isotropic clearing temperature of the LCE, the pattern painted on the bottom layer of the film showed up. (c) The pattern disppeared when the film was cooled down to room temperature. Scale bar: 1 cm. 'r.t.' represents room temperature. 


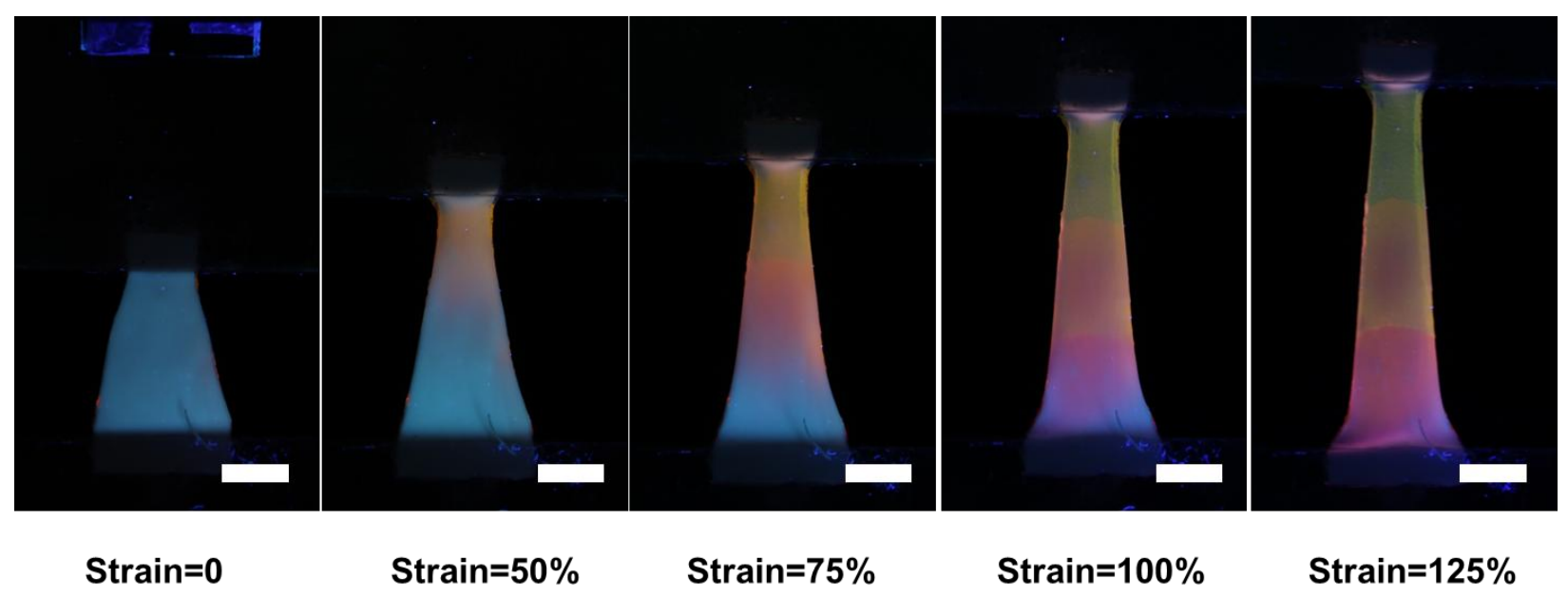

Figure 7. The photos of trapezoid mechanochromic film with a tricolor pattern painted on the bottom layer of the film. When the applied average strain of the film was larger than $50 \%$, the region of the film with narrowest width shows yellow color. Stretching the film to $75 \%$ average strain led to the appearance of both yellow and orange. Further increase of the strain to $100 \%$ resulted in the appearance of the entire tricolor pattern. Scale bar in the figure is $1 \mathrm{~cm}$. 
(a)
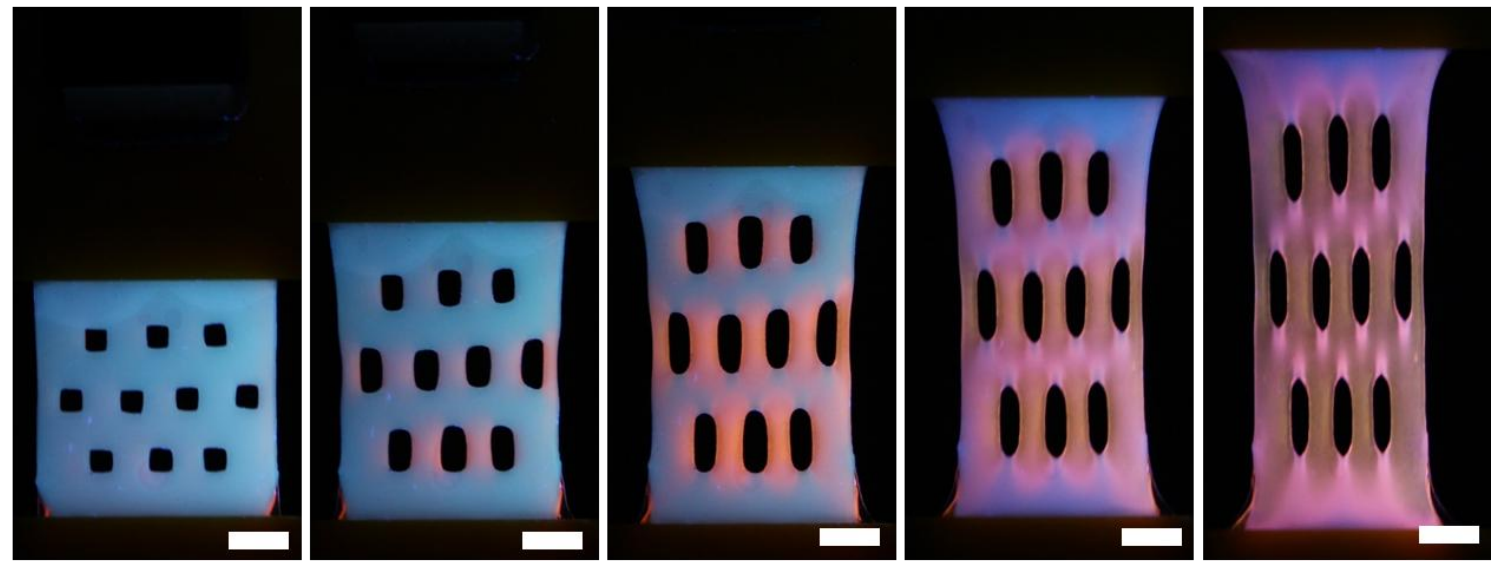

(b)
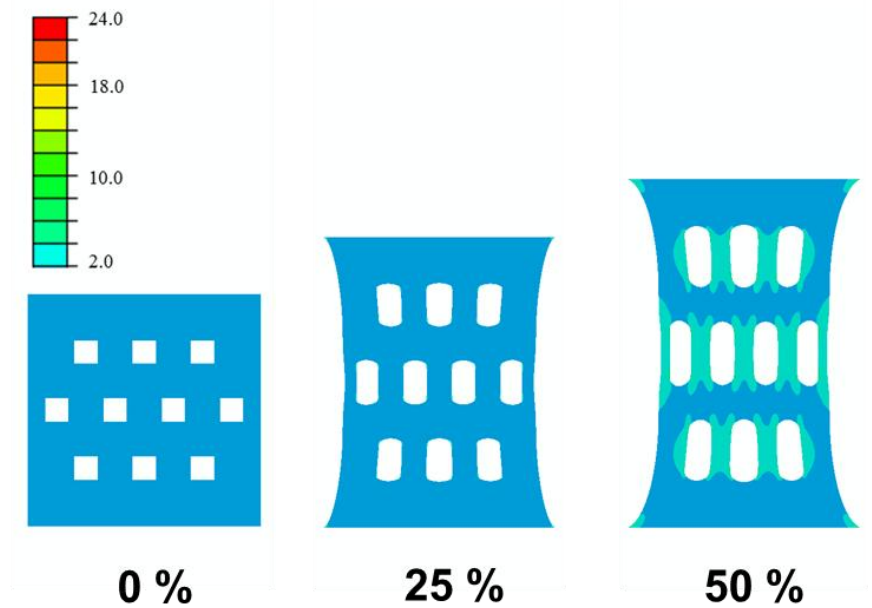

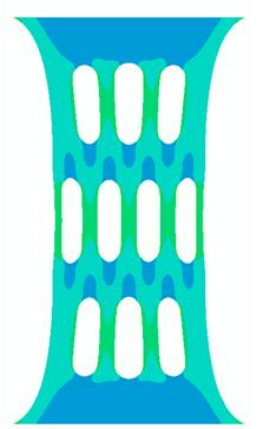

$75 \%$

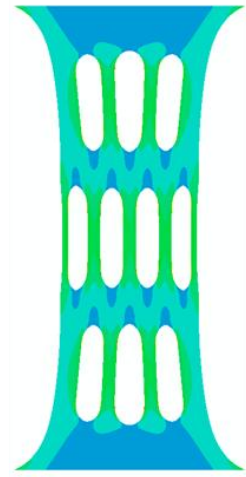

$100 \%$

Figure 8. (a) Complex patterns developed in the fabricated mechanochromic film with periodically distributed square holes and subjected to unaxial stretch. (b) Finite-element simulations of the mechanochromic film at different applied strains. The color in the figure stands for the first invariant of Cauchy-Green strain tensor in the film. 